\title{
ПАРАМЕТРЫ ФАРМАКОКИНЕТИКИ ВАНКОМИЦИНА У БОЛЬНЫХ С НАРУШЕНИЕМ ФУНКЦИИ ПОЧЕК В ПОСЛЕОПЕРАЦИОННОМ ПЕРИОДЕ: СРАВНЕНИЕ РЕЗУЛЬТАТОВ ФАРМАКОКИНЕТИЧЕСКОГО ИССЛЕДОВАНИЯ И МАТЕМАТИЧЕСКОГО МОДЕЛИРОВАНИЯ
}

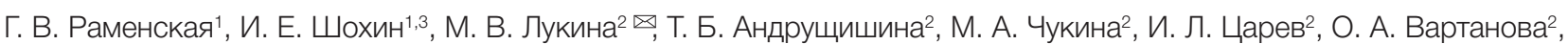

Т. Е. Морозова

Кафедра фармацевтической и токсикологической химии имени А. П. Арзамасцева, Институт фармации,

Первый Московский государственный медицинский университет имени И. М. Сеченова (Сеченовский Университет), Москва

Кафедра клинической фармакологии и пропедевтики внутренних болезней, лечебный факультет,

Первый Московский государственный медицинский университет имени И. М. Сеченова (Сеченовский Университет), Москва

ООО "Центр Фармацевтической Аналитики", Москва

В клинической практике возможной альтернативой фармакокинетическим исследованиям (ФКИ) является методика математического моделирования (ММ) фармакокинетических (ФК) и фармакодинамических (ФД) параметров для расчета доз антибактериальных препаратов. Целью исследования было сравнение параметров ФК ванкомицина, полученных на основе ФКИ и ММ, у пациентов с нарушением функции почек в послеоперационном периоде. В проспективное исследование был включен 61 пациент (47 мужчин и 14 женщин, возраст 60,59 12,23 лет). В ходе ФКИ методом высокоэффективной жидкостной хроматограффии определяли $C_{\text {trun }}, C_{\text {peка }}$, с последующим расчетом площади под фармакокинетической кривой (ПФК ${ }_{24}$ ). Расчет параметров ФК при ММ проводили с помощью программы R 3.4.0 на основе однокомпартментной модели. По данным ФКИ значения равновесных $C \quad 4 е р е з 48$ ч от начала антибактериальной терапии были достоверно ниже значений, полученных при МM ( $p=0,004)$. В группе пациентов с острым почечным повреждением (ОПП) на момент завершения терапии значения ПФК 24 по данным ФКИ были достоверно выше $(p=0,011)$. Вероятность достижения целевого отношения ПФК $24 / \mathrm{MПK}_{24} 400$ мКг•ч/мл выше в группе пациентов, где $C_{\text {trough }}$ составляет 10-15 мкг/мл. Таким образом, результаты исследования подтверждают, что у больных с нарушением функции почек в послеоперационном периоде применение ММ имеет ряд ограничений и необходимо проведение терапевтического лекарственного мониторинга (ТЛМ).

Ключевые слова: фармакокинетическое исследование, фармакокинетика ванкомицина, математическое моделирование, острое почечное повреждение, пациенты хирургического профиля

Благодарности: авторы благодарят Бабенко Олега Васильевича, главного врача УКБ № 1 Первого МГМУ им. И. М. Сеченова, за предоставленную возможность проведения фармакокинетического исследования.

$\triangle$ Для корреспонденции: Мария Владимировна Лукина

ул. Большая Пироговская, 2, стр. 4, Москва, 119435; mari-luk2010@yandex.ru

Статья получена: 16.05.2018 Статья принята к печати: 25.08.2018

DOI: $10.24075 / \mathrm{vrgmu} .2018 .051$

\section{PARAMETERS OF VANCOMYCIN PHARMACOKINETICS IN POSTOPERATIVE PATIENTS WITH RENAL DYSFUNCTION: COMPARING THE RESULTS OF A PHARMACOKINETIC STUDY AND MATHEMATICAL MODELING}

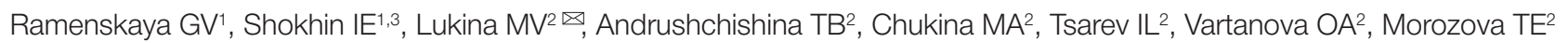

Department of Pharmaceutical and Toxicological Chemistry, Institute of Pharmacy,

Sechenov First Moscow State Medical University (Sechenov University), Moscow

Department of Clinical Pharmacology and Propaedeutics of Internal Diseases, Faculty of General Medicine

Sechenov First Moscow State Medical University (Sechenov University), Moscow

Center of Pharmaceutical Analytics Ltd., Moscow

Mathematical modeling of pharmacokinetic (PK) and pharmacodynamic (PD) parameters essential for establishing correct dosing regimens is an alternative to pharmacokinetic studies (PKS) adopted in the clinical setting. The aim of this work was to compare the values of PK parameters for vancomycin obtained in an actual PKS and through MM in postoperative patients with kidney injury. Our prospective study included 61 patients (47 males and 14 females aged $60.59 \pm 12.23$ years). During PKS, drug concentrations at steady state $C_{\text {trough }}$ and $C_{\text {peak }}$ were measured by high-performance liquid chromatography followed by the

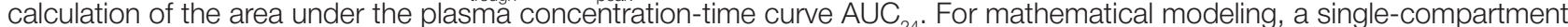
model was employed; PK parameters were estimated using R 3.4.0. The values of Ctrough measured $48 \mathrm{~h}$ after the onset of antibiotic therapy during PKS were significantly lower than those predicted by MM $(p=0.004)$. In a group of patients with acute kidney injury (AKI), $\mathrm{AUC}_{24}$ measured at the end of treatment was significantly higher than its value predicted by $\mathrm{MM}$ $(p=0.011)$. The probability of achieving the target $A_{U C}$ to $\mathrm{MIC}$ ratio of over $400 \mu \mathrm{g} \cdot \mathrm{h} / \mathrm{ml}$ is higher in the group of patients with $C_{\text {troun }}=10-15 \mu \mathrm{g} / \mathrm{ml}$. Our findings confirm that the use of MM in postoperative patients with renal dysfunction is limited and therapeutic drug monitoring should be used instead.

Keywords: pharmacokinetic study, vancomycin pharmacokinetics, mathematical modeling, acute kidney injury, surgical patients Acknowledgements: the authors wish to thank Oleg V. Babenko, Chief Physician of the University Clinical Hospital No.1 of Sechenov First Moscow State Medical University for providing an opportunity to carry out our research

$\triangle$ Correspondence should be addressed: Maria V. Lukina

Bolshaya Pirogovskaya, 2, bl. 4, Moscow, 119435; mari-luk2010@yandex.ru

Received: 16.05.2018 Accepted: 25.08.2018

DOI: 10.24075/brsmu.2018.051 
В настоящее время при назначении антибактериальных препаратов с узким терапевтическим диапазоном для проведения эффективной и безопасной терапии возможно проведение фармакокинетического исследования (ФКИ) или терапевтического лекарственного мониторинга (ТЛМ). Согласно международным рекомендациям, в рамках ТЛМ ванкомицина необходимо проводить измерение равновесных остаточных концентраций $\left(\mathrm{C}_{\text {trough }}\right)$, расчет площади под фармакокинетической кривой за 24 ч (ПФК 24$)$ и определять отношения ПФК ${ }_{24}$ к минимальной подавляющей концентрации (МПК). Существует ряд ограничений, не позволяющих активно использовать ТЛМ в рутинной практике, зачастую связанных с невозможностью получения большого количества образцов крови для расчета ПФК ${ }_{24}[1,2]$.

Применение математического моделирования (ММ) параметров ФК антибактериальных препаратов может быть альтернативой ТЛМ в отдельных клинических ситуациях. Для выбора стартового режима дозирования некоторых антибактериальных препаратов (ванкомицина, аминогликозидов, колистина) были предложены различные варианты ММ, или «медицинские калькуляторы» [3, 4]. Для ванкомицина «медицинский калькулятор» основан на однокомпартментной фармакокинетической модели и позволяет проводить моделирование отношения параметров ФК/ФД для расчета доз препарата с учетом индивидуальных особенностей пациента (пол, возраст, вес, почечная функция), значения МПК [5, 6]. Внедрение в клиническую практику различных вариантов ММ, дает возможность снизить потребность в проведении ТЛМ.

Сведения о возможности ММ параметров ФК в отдельных группах пациентов немногочисленны. Кроме того, существует ряд недостатков, связанных с невозможностью оценки динамики процессов биотрансформации, объема распределения и скорости элиминации антибактериальных препаратов у пациентов с острыми нарушениями функции почек в раннем послеоперационном периоде. Имеют значение также высокая стоимость оборудования и программного обеспечения, необходимого для проведения ММ $[7,8]$.
Анализ литературы не позволяет сделать однозначного вывода о возможности замены ТЛМ методами ММ, поскольку ограничены данные о сопоставимости результатов этих методик в различных клинических ситуациях.

Таким образом, для совершенствования методики ММ параметров ФК необходимо проведение ФКИ в различных группах пациентов. Данные исследования позволят оптимизировать эффективность и безопасность терапии ванкомицином в отдельных группах пациентов.

Целью работы было сравнение параметров ФК ванкомицина, полученных при ФКИ и с помощью ММ у пациентов хирургического профиля с острым нарушением функции почек.

\section{ПАЦИЕНТЫ И МЕТОДЬ}

Проспективное обсервационное исследование было проведено на базе Университетской клинической больницы №1 Первого МГМУ им. И. М. Сеченова в период с сентября 2016 г. по январь 2018 г. Протокол исследования был одобрен локальным этическим комитетом (№ 05-16 от 18.05.2016).

В исследование включен 61 пациент (из них 47 мужчин и 14 женщин) с гнойно-септическими осложнениями после оперативного вмешательства. Средний возраст составил $60,59 \pm 12,23$ года. В послеоперационном периоде пациенты были разделены на 2 группы в зависимости от наличия острого почечного повреждения (ОПП) [9]: 1-я группа - пациенты с ОПП ( $n=35 ; 66,6 \%), 2$-я группа - без ОПП (контрольная группа) ( $n=26 ; 33,4 \%)$. В группе с ОПП преобладали пациенты с легким и умеренным нарушением функции почек: 1-я стадия ОПП была диагностирована у 19 (31,1\%) пациентов, 2-я стадия у 13 пациентов (21,3\%), 3-я стадия у 3 пациентов (4,9\%) Подробная характеристика пациентов, включенных в исследование, представлена в табл. 1. Группы были сравнимы по основным клиническим характеристикам, однако в группе с ОПП пациенты были достоверно старше ( $p=0,004)$. В послеоперационном

Таблица 1. Клиническая характеристика пациентов, включенных в исследование

\begin{tabular}{|c|c|c|c|c|}
\hline \multirow{2}{*}{ Клиническая характеристика } & Итого $n=61$ & Без ОПП $n=26$ (44,8\%) & С ОПП $n=35$ (55,7\%) & \multirow{2}{*}{$p$} \\
\hline & $M \pm S D$ & $M \pm S D$ & $M \pm S D$ & \\
\hline Возраст, лет & $60,59 \pm 12,23$ & $55,46 \pm 12,89$ & $64,4 \pm 10,33$ & $0,004^{*}$ \\
\hline ИМТ, кг/м² & $27,4 \pm 5,2$ & $27,12 \pm 6,1$ & $27,29 \pm 4,5$ & 0,726 \\
\hline$\Phi \mathrm{B}_{0}, \%$ & $59,02 \pm 7,86$ & $62,53 \pm 6,74$ & $56,89 \pm 7,83$ & $0,018^{*}$ \\
\hline $\mathrm{KK}_{0}$, мл/мин & $96,48 \pm 29,01$ & $96,26 \pm 24,76$ & $96,64 \pm 32,16$ & 0,96 \\
\hline $\mathrm{KK}_{1}$, мл/мин & $61,5 \pm 27,2$ & $81,51 \pm 23,54$ & $46,64 \pm 19,1$ & $<0,0001^{*}$ \\
\hline $\mathrm{KK}_{2}$ мл/мин & $85,98 \pm 32,33$ & $87,37 \pm 33,52$ & $84,95 \pm 31,86$ & 0,776 \\
\hline Альбумин ${ }_{0}$, мг/дл & $41,21 \pm 4,2$ & $42,46 \pm 4,35$ & $40,29 \pm 3,97$ & 0,447 \\
\hline Альбумин ${ }_{1}$, мг/дл & $33,56 \pm 1,52$ & $32,21 \pm 2,84$ & $44,57 \pm 1,61$ & $0,047^{*}$ \\
\hline Длительность госпитализации, сут. & $25,07 \pm 15,069$ & $26,77 \pm 4,27$ & $23,8 \pm 1,17$ & 0,451 \\
\hline Длительность ИВЛ, сут. & $3,30 \pm 1,75$ & $3,00 \pm 1,29$ & $3,51 \pm 0,887$ & 0,736 \\
\hline Длительность ОРИТ, сут. & $6,46 \pm 1,187$ & $6,5 \pm 2,27$ & $6,43 \pm 1,23$ & 0,977 \\
\hline Кровопотеря, мл & $653,44 \pm 604,65$ & $512,1 \pm 258,8$ & $758,00 \pm 754,66$ & 0,118 \\
\hline Летальность, \% & $11(18 \%)$ & $4(36,4 \%)$ & $7(63,6 \%)$ & 0,454 \\
\hline
\end{tabular}

Примечание: * - достоверные различия, $p_{\text {ve }}<$ 0,05; ИМТ — индекс массы тела; КК — клиренс креатинина по Кокрофту-Голту; КК - до операции; $\mathrm{KK}_{1}$ - на 2-3 сутки после операции; $\mathrm{KK}_{2}$ - на 7-10 сутки после операции; ИВЛ — искусственная вентиляция легких; ФВ - фракция выброса. 
периоде в группе с ОПП уровень альбумина у больных был выше, чем в группе сравнения ( $p=0,047)$.

\section{Режим назначения ванкомицина}

Все пациенты с инфекционными осложнениями получали ванкомицин (Эдицин) (Сандоз; Словения). Режим дозирования ванкомицина определяли в соответствии с инструкцией по медицинскому применению из расчета 15-20 мг/кГ массы тела (максимальная суточная доза не превышала 2 г) с учетом функции почек на основе расчетных значений клиренса креатинина (КК, мл/мин) по формуле Кокрофта Голта. Ванкомицин вводили внутривенно капельно в течение 60 мин каждые 12 ч [10]. Последующая коррекция режима дозирования проводилась через 24-48 ч с учетом расчетного значения КK.

Пациенты с ОПП получали достоверно более низкие суточные дозы ванкомицина, чем пациенты без нарушений функции почек $(928,6 \pm 275$ мг и 1637,9 \pm 515,8 мг соответственно; $p<0,0001)$. Длительность антибактериальной терапии зависела от тяжести инсекционных осложнений, локуса, результатов микробиологического исследования и индивидуальной переносимости терапии и составила 9,61 \pm 3,8 сут. Достоверно длительность терапии в группах не различалась $(9,17 \pm 3,6$ и 10,19 \pm 4 сут. соответственно; $p=0,353)$.

\section{Параметры фармакокинетики ванкомицина по данным фармакокинетического исследования на основе высокоэффективной жидкостной хроматографии}

Всем пациентам, включенным в исследование, проводили забор образцов крови для ФКИ в соответствии с рекомендациями по ТЛМ ванкомицина [1]. Для определения равновесных концентраций $C_{\text {peak }}$ (через 60 мин после окончания внутривенной инфузии) и $C_{\text {trough }}$ (за 60 мин перед введением очередной дозы препарата) взятие образцов крови проводили через 48 ч от начала (1) и на момент завершения антибактериальной терапии (2) [11].

Подготовку образцов проводили путем осаждения белков метанолом. Количественное определение проводили на высокоэффективном жидкостном хроматограсре Agilent 1260, оснащенном градиентным насосом, дегазатором, автосамплером и тандемным масс-селективным детектором Agilent 6460 (AgilentTechnologies; США). Хроматографическое разделение проводили на колонке ZorbaxEclipse Plus-C18 2,1 × 50 мм, 1,8 мкм с предколонкой Zorbax Eclipse Plus C18 12,5 × 2,1 мм, 1,8 мкм.

На основании полученных равновесных значений $C_{\text {peak }}$ и $C_{\text {trough }}$ проводили расчет ПФК ${ }_{24}$ с помощью уравнений динамики как сумму различных фаз («метод трапеций») [12]:

$$
\Pi \Phi \mathrm{K}_{24}=\frac{(\text { Lintrap }+ \text { Logtrap }) \times 24}{\tau} ;
$$

где Lintrap - площадь под фармакокинетической кривой линейной фазы инфузии:

$$
\text { Lintrap }=\frac{\left(C_{\text {trough }}+C_{\text {peak }}\right) \times T_{\text {inf }}}{2} ;
$$

где $T_{\text {inf }}$ - продолжительность инфузии (ч).

Logtrap - площадь под «логарифмической» фазой элиминации:

$$
\text { Logtrap }=\frac{\left(C_{\text {peak }}-C_{\text {trough }}\right) \tau-T_{\text {inf }}}{\ln C_{\text {peak }}^{C_{\text {trough }}}} ;
$$

где $\tau$ - время между инфузиями (ч)

\section{Методика проведения математического моделирования}

Математическое моделирование проводили с использованием компьютерной программы R 4.3.0 [12]. Определяли расчетные значения $C_{\text {peak }}, C_{\text {trough }}$ и ПФК 24 с использованием уравнений «динамики» для однокомпартментной модели через 48 ч от начала (1) и на момент завершения антибактериальной терапии (2) [13] по формулам:

$$
\begin{gathered}
C_{\text {peak }}=\frac{\text { Dose } \times 1-e^{-T_{\text {int }} \times K_{\text {el }}}}{T_{\text {inf }} \times V_{d} \times K_{\text {el }} \times\left(1-e^{-\tau \times K_{\text {ed }}}\right)} ; \\
C_{\text {trough }}=C_{\text {peak }} \times e^{-K_{\text {el }} \times\left(\tau-T_{\text {ind }}\right)} ;
\end{gathered}
$$

где Dose - разовая доза ванкомицина (мг),

$T_{\text {inf }}$ - продолжительность инфузии (ч),

$\tau$ - время между инфузиями (ч),

$K_{\text {el }}$ - предсказанная константа элиминации $\left(4^{-1}\right)$,

$V_{d}^{\text {el }}$ - кажущийся объем распределения (л/кг):

$$
V_{d}=0,7 \times M
$$

где $M$ - абсолютная масса тела пациента (кг).

Для расчета предсказанной константы элиминации применяли следующее уравнение [14]:

$$
K_{\text {el }}=0,00083 \times K K+0,0044 ;
$$

где КК — клиренс креатинина (мл/мин) по Кокрофту-Голту: $\mathrm{KK}=\frac{[140-\text { возраст] } \times \text { вес тела }(\text { Кг) } \times(10,05 \text { для жен или 10,23 для муж) }}{\text { креатинин плазмы крови (мкмоль/л) }}$.

Для расчета ПФК 24 применяли «метод трапеции»:

$$
\Pi Ф \mathrm{~K}_{24}=\frac{(\text { Lintrap }+ \text { Logtrap }) \times 24}{\tau} .
$$

Статистический анализ проводили с помощью пакета прикладных программ IBMSPSS Statistics 18.0. и R 3.4.0. Данные представлены в виде средних величин (M) и среднего квадратичного отклонения (SD) для нормально распределенных непрерывных величин; медианы (Me) и интерквартильного размаха (IQR) для остальных случаев и частот для категориальных переменных. Нормальность распределения проверяли с помощью теста Шапиро-Уилка. Достоверность различий частот определяли при помощи точного критерия Фишера. Достоверность различий среднего арифметического в группах определяли при помощи дисперсионного анализа (ANOVA). Помимо дисперсионного анализа, использовали непараметрические методы, определяли достоверность различия средних рангов между двумя группами с помощью U-критерия Манна-Уитни-Уилкоксона. Различия считали статистически достоверными (статистически значимыми) при $p<0,05$. Для установления корреляционных связей между клинически значимыми параметрами фармакокинетики, $C_{\text {trough }}$ и ПФК ${ }_{24}$, использовали корреляционный анализ по Спирмену.

\section{РЕЗУЛЬТАТЫ ИССЛЕДОВАНИЯ}

При сравнении параметров ФК ванкомицина по данным ФКИ и ММ через 48 ч от начала (1) и на момент завершения антибактериальной терапии (2) было установлено, что фактические значения $K_{\text {el }}{ }^{1}$ были достоверно выше расчетных значений по данным MM $(0,109(0,08-0,15)$ и 0,06 (0,04-0,072) соответственно; $p<0,0001)$. Фактические значения равновесных $C_{\text {trough }}{ }^{1}$ были достоверно ниже 
значений, полученных при ММ $(11,32(8,1-16,4)$ и 16,59 $(14,03-24,8)$ соответственно; $p=0,004)$. При этом значения $C_{\text {trough }}{ }^{2}$ по данным ФКИ и ММ достоверно не различались. Через 48 ч от начала антибактериальной терапии значения ПФК ${ }_{24}$ достоверно не различались $(p=0,715)$. На момент завершения антибактериальной терапии фактические значения ПФК ${ }_{24}^{2}$ были достоверно выше расчетного уровня $(564,04(409,5-751,9)$ и $347,03 \quad(267,43-479,99)$ соответственно; $p=0,011$ ) (табл. 2).

При сравнении параметров ФК по данным ФКИ и ММ в группах с ОПП и без ОПП достоверных различий не получено, за исключением фактических значений уровня $K_{\text {el }}{ }^{1}(p=0,037)$, которая была достоверно выше у пациентов с нарушениями функции почек (табл. 2).
Параметры ФК ванкомицина, полученные в ходе ФКИ, демонстрируют высокую вариабельность значений $C_{\text {trough }}$ и ПФК ${ }_{24}$ как на момент начала, так и по завершении антибактериальной терапии (рис. 1). Это можно объяснить индивидуальными особенностями ФК ванкомицина в представленной выборке на фоне стандартного режима дозирования. Однако полученный разброс ФК параметров ванкомицина по данным ММ и достоверное отличие от фактических результатов ФКИ ограничивают возможность применения данного варианта ММ в группе пациентов с острыми нарушениями функции почек.

В группе пациентов, у которых уровень равновесных $C_{\text {trough }}$ достигал 10-15 мкг/мл, значения ПФК 24 были выше 400 мкг × ч /мл как через 48 ч от начала терапии (рис. 2),

Таблица 2. Сравнение параметров фармакокинетики ванкомицина, полученных в результате проведения ФКИ и ММ, в том числе в группах больных о ОПП и без ОПП, через 48 ч от начала терапии и на момент завершения терапии

\begin{tabular}{|c|c|c|c|c|c|c|c|c|c|}
\hline \multirow{3}{*}{$\begin{array}{c}\text { ФК } \\
\text { показатель }\end{array}$} & \multirow{2}{*}{$\begin{array}{c}\text { ТЛМ } \\
(n=61)\end{array}$} & \multirow{2}{*}{$\begin{array}{c}\mathrm{MM} \\
(n=61)\end{array}$} & \multirow{2}{*}{$\begin{array}{c}\text { Тест } \\
\text { Манна- } \\
\text { Уитни- } \\
\text { Уилкоксона; } \\
p\end{array}$} & \multicolumn{2}{|c|}{ ТЛМ $(n=61)$} & \multirow{2}{*}{$\begin{array}{c}\text { Тест } \\
\text { Манна- } \\
\text { Уитни- } \\
\text { Уилкоксона; } \\
p\end{array}$} & \multicolumn{2}{|c|}{$\mathrm{MM}(n=61)$} & \multirow{2}{*}{$\begin{array}{c}\text { Тест } \\
\text { Манна- } \\
\text { Уитни- } \\
\text { Уилкоксона; } \\
p\end{array}$} \\
\hline & & & & $\begin{array}{c}\text { ОПП+ } \\
(n=35)\end{array}$ & ОПП- & & ОПП+ & ОПП- & \\
\hline & \multicolumn{3}{|c|}{$\mathrm{Me}[\mathrm{IQR}]$} & \multicolumn{3}{|c|}{$\mathrm{Me}[\mathrm{IQR}]$} & \multicolumn{3}{|c|}{$\mathrm{Me}[\mathrm{IQR}]$} \\
\hline $\operatorname{Kel}^{1}\left(4^{-1}\right)$ & $\begin{array}{c}0,109 \\
{[0,08-0,15]}\end{array}$ & $\begin{array}{c}0,06 \\
{[0,04-0,072]}\end{array}$ & $<0,0001$ & $\begin{array}{c}0,12 \\
{[0,1-0,14]}\end{array}$ & $\begin{array}{c}0,1 \\
{[0,06-0,131]}\end{array}$ & 0,037 & $\begin{array}{c}0,04 \\
{[0,04-0,07]}\end{array}$ & $\begin{array}{c}0,06 \\
{[0,06-0,077]}\end{array}$ & 0,117 \\
\hline $\mathrm{Kel}^{2}\left(4^{-1}\right)$ & $\begin{array}{c}0,08 \\
{[0,05-0,14]}\end{array}$ & $\begin{array}{c}0,08 \\
{[0,063-0,102]}\end{array}$ & 0,274 & $\begin{array}{c}0,06 \\
{[0,05-0,15]}\end{array}$ & $\begin{array}{c}0,11 \\
{[0,07-0,13]}\end{array}$ & 0,412 & $\begin{array}{c}0,08 \\
{[0,05-0,15]}\end{array}$ & $\begin{array}{c}0,09 \\
{[0,07-0,11]}\end{array}$ & 0,709 \\
\hline $\begin{array}{l}\mathrm{C}_{\text {trough }}{ }^{1} \\
\text { (МКГ/МЛ) }\end{array}$ & $\begin{array}{c}11,32 \\
{[8,1-16,4]}\end{array}$ & $\begin{array}{c}16,59 \\
{[14,03-24,8]}\end{array}$ & 0,004 & $\begin{array}{c}9,6 \\
{[6,9-15,0]}\end{array}$ & $\begin{array}{c}12,08 \\
{[8,8-18,27]}\end{array}$ & 0,197 & $\begin{array}{c}16,2 \\
{[14,2-19,7]}\end{array}$ & $\begin{array}{c}14,03 \\
{[13,24-18,04]}\end{array}$ & 0,54 \\
\hline $\begin{array}{l}\mathrm{C}_{\text {trough }}{ }^{2} \\
\text { (МКГ/MЛ) }\end{array}$ & $\begin{array}{c}12,59 \\
{[8,5-22,8]}\end{array}$ & $\begin{array}{c}8,65 \\
{[5,9-12,06]}\end{array}$ & 0,092 & $\begin{array}{c}15,7 \\
{[6,6-25,8]}\end{array}$ & $\begin{array}{c}12,59 \\
{[9,1-21,7]}\end{array}$ & 0,776 & $\begin{array}{c}8,3 \\
{[6,08-11,6]}\end{array}$ & $\begin{array}{c}10,14 \\
{[5,7-12,5]}\end{array}$ & 0,765 \\
\hline $\begin{array}{l}\mathrm{C}_{\text {peak }}{ }^{1} \\
\text { (МКГ/MЛ) }\end{array}$ & $\begin{array}{c}35,6 \\
{[31,2-37,2]}\end{array}$ & $\begin{array}{c}27,3 \\
{[24,2-32,2]}\end{array}$ & 0,019 & $\begin{array}{c}35,1 \\
{[30,9-37,8]}\end{array}$ & $\begin{array}{c}23,8 \\
{[21,3-31,4]}\end{array}$ & 0,502 & $\begin{array}{c}26,2 \\
{[15,8-27,2]}\end{array}$ & $\begin{array}{c}28,2 \\
{[26,6-32,8]}\end{array}$ & 0,502 \\
\hline $\begin{array}{l}\mathrm{C}_{\text {peak }}{ }^{2} \\
\text { (МК/MЛ) }\end{array}$ & $\begin{array}{c}22,5 \\
{[18,6-30,7]}\end{array}$ & $\begin{array}{c}34,8 \\
{[31,7-41,9]}\end{array}$ & 0,002 & $\begin{array}{c}35,6 \\
{[31,9-40,7]}\end{array}$ & $\begin{array}{c}23,8 \\
{[21,3-31,4]}\end{array}$ & 0,263 & $\begin{array}{c}26,23 \\
{[24,11-28,1]}\end{array}$ & $\begin{array}{c}34,8 \\
{[30,1-43,1]}\end{array}$ & 0,263 \\
\hline $\begin{array}{l}\text { ПФК }{ }_{24}{ }^{1} \\
\left(\mathrm{MK \digamma}^{*} 4 / \mathrm{M}\right)\end{array}$ & $\begin{array}{c}484,08 \\
{[404,5-604,4]}\end{array}$ & $\begin{array}{c}459,72 \\
{[433,6-556,01]}\end{array}$ & 0,715 & $\begin{array}{c}465,7 \\
{[399,5-605,3]}\end{array}$ & $\begin{array}{c}530,8 \\
{[480,2-603,4]}\end{array}$ & 0,263 & $\begin{array}{c}462,8 \\
{[450,4-548,5]}\end{array}$ & $\begin{array}{c}458,38 \\
{[413,8-553,5]}\end{array}$ & 0,709 \\
\hline $\begin{array}{l}\text { ПФК }{ }_{24}^{2} \\
\left(\text { МКГ* }{ }^{*} 4 / \mathrm{M}\right)\end{array}$ & $\begin{array}{c}564,04 \\
{[409,5-751,9]}\end{array}$ & $\begin{array}{c}347,03 \\
{[267,43-479,99]}\end{array}$ & 0,011 & $\begin{array}{c}551,2 \\
{[397,02-786,6]}\end{array}$ & $\begin{array}{c}564,04 \\
{[421,9-721,58]}\end{array}$ & 0,765 & $\begin{array}{c}345,4 \\
{[255,5-393,2]}\end{array}$ & $\begin{array}{c}386,8 \\
{[273,8-481,5]}\end{array}$ & 0,502 \\
\hline
\end{tabular}

Примечание: 1 - через 48 ч от начала терапии; 2 - на момент завершения терапии.

Через 48 ч после начала АБТ (первая проба)

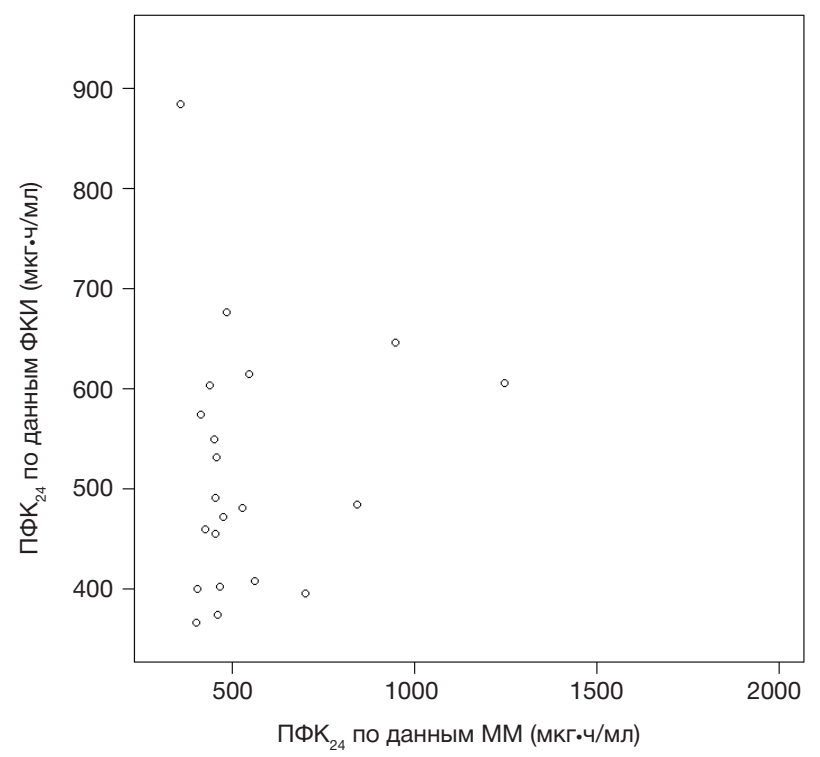

На момент завершения АБТ (вторая проба)

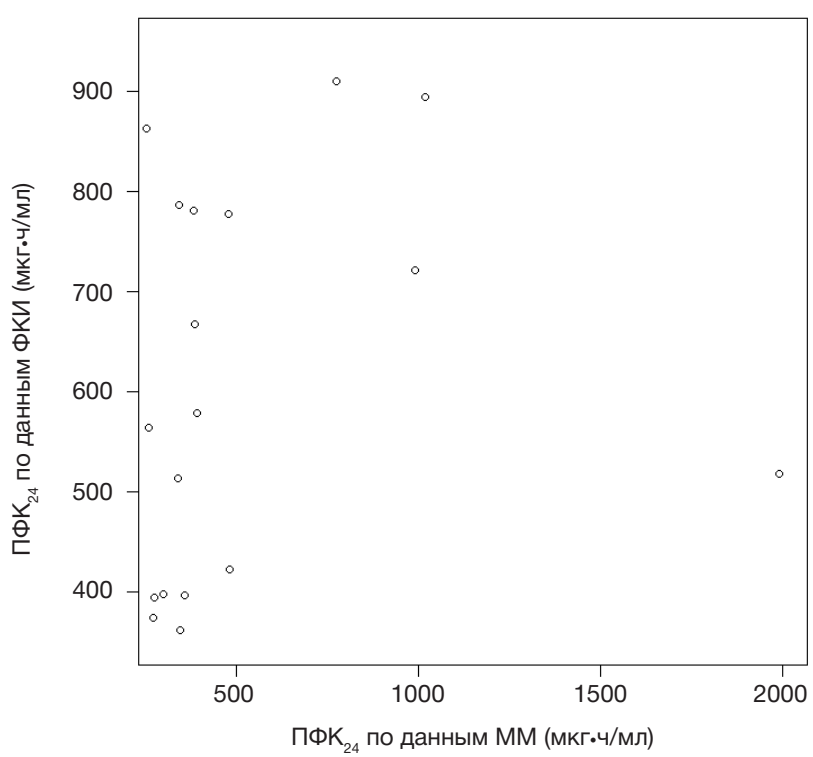

Рис. 1. Разброс значений ПФК периоде через 48 ч и на момент завершения терапии ванкомицином 
так и на момент завершения антибактериальной терапии (рис. 3). По данным корреляционного анализа была получена положительная корреляционная связь между значениями равновесных $C_{\text {trough }}$ и ПФК ${ }_{24}(r=0,964 ; p<0,001)$.

\section{Прогнозирование вероятности достижения целевого отношения ФК/ФД}

Полученные значения ПФК 24 в ходе ФКИ свидетельствуют о высокой вероятности достижения целевого отношения ФК/ФД (ПФК24/МПК > 400) в случае, если МПК = 1 мКГ/Мл для Staphylococcus aureus. Исключение составляет группа пациентов, в которой значение равновесных $C_{\text {trough }}$ было ниже 10 мкг/мл; в этом случае целевое отношение ФК ФД было отмечено у 55\% пациентов. В случае увеличения значений МПК до 1,5 и 2 мкг/Мл вероятность достижения целевого отношения ФК/ФД в группе пациентов, где $C_{\text {trough }}$ был 10-15 мкг/мл, снижается до 30\%, в группе, где $C_{\text {trough }}$ составлял 15-20 мкг/мл - снижается до 70\% (табл. 3). Гипотетически целевое отношение ФК/ФД при увеличении МПК до 2 мкг/мл возможно лишь в случае достижения $C_{\text {trough }} 20$ мкг/мл и выше (табл. 3).

При сравнении результатов прогнозирования отношения $П Ф \mathrm{~K}_{24} / \mathrm{MПK}$ у пациентов на момент завершения антибактериальной терапии, целевых значений ФК/ФД (> 400) в большинстве случаев достигали пациенты, у которых уровень $C_{\text {trough }}$ был выше 10-15 мкг/мл (табл. 4).

\section{ОБСУЖДЕНИЕ РЕЗУЛЬТАТОВ}

В ходе исследования было выявлено, что при стандартном подходе к дозированию ванкомицина у пациентов хирургического профиля с острыми нарушениями функции почек фактические значения $C_{\text {trough }}$ через 48 ч

Через 48 ч после начала АБТ (первая проба)

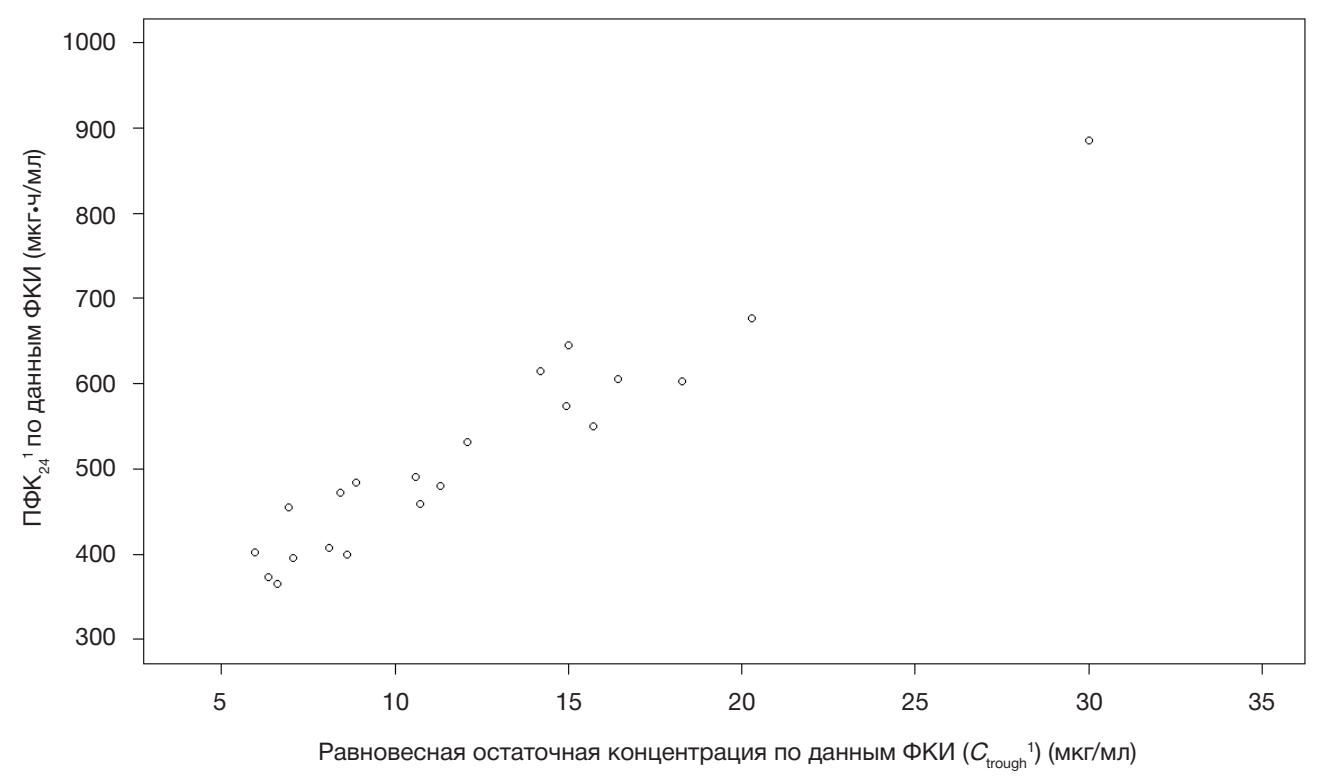

Рис. 2. Зависимость значения $П Ф \mathrm{~K}_{24}{ }^{1}$ от уровня равновесной $C_{\text {trough }}{ }^{1}$ через 48 ч от начала антибактериальной терапии по данным ФКИ

На момент завершения АБТ (вторая проба)

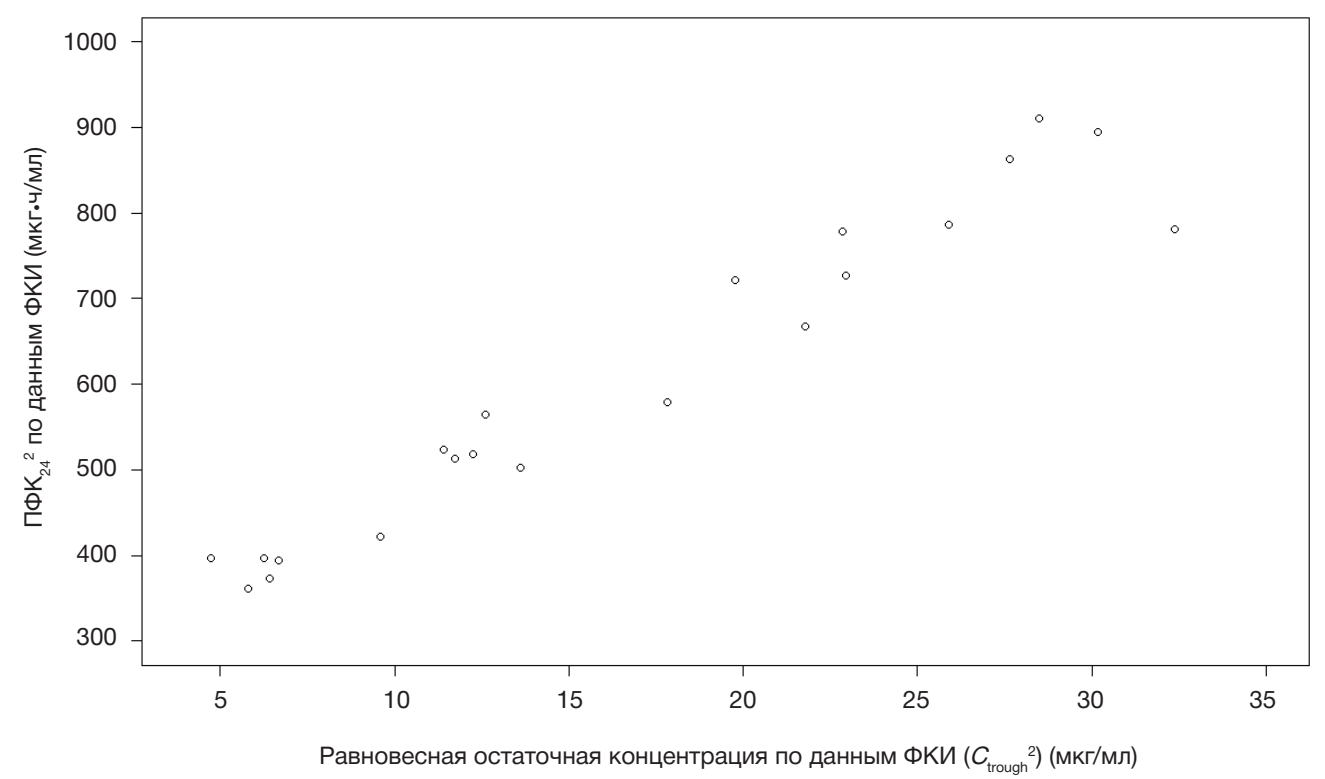

Рис. 3. Зависимость значения ПФК ${ }_{24}{ }^{2}$ от уровня равновесной $C_{\text {trough }}{ }^{2}$ на момент завершения антибактериальной терапии по данным ФКИ 
Таблица 3. Прогнозирование отношения ПФК ${ }_{24} / \mathrm{MПK}$ для Staphylococcus aureus через 48 ч от начала терапии ванкомицином

\begin{tabular}{|c|c|c|c|c|c|c|}
\hline \multirow{2}{*}{ Значение $C_{\text {trough }}$, Мкг/мл } & \multicolumn{3}{|c|}{ ПФК ${ }_{24}$, мкГ.ч/МЛ } & \multicolumn{3}{|c|}{$\Pi \Phi \mathrm{K}_{24} / \mathrm{M} \Pi \mathrm{K}>400$} \\
\hline & M & $\min$ & $\max$ & МПК 1 мкг/мл (\%) & МПК 1,5 мкг/мл (\%) & МПК 2 мкг/мл (\%) \\
\hline$<10$ & 401,9753 & 365,676 & 484,0849 & 55 & 0 & 0 \\
\hline $10-15$ & 530,8875 & 459,4124 & 645,6017 & 100 & 30 & 0 \\
\hline $15-20$ & 603,4062 & 549,4891 & 605,2955 & 100 & 70 & 0 \\
\hline$>20$ & 780,6152 & 676,4806 & 884,7498 & 100 & 100 & 50 \\
\hline
\end{tabular}

Таблица 4. Прогнозирование отношения ПФК ${ }_{24}$, мкг 4 /мл для Staphylococcus aureus на момент завершения терапии ванкомицином

\begin{tabular}{|c|c|c|c|c|c|c|}
\hline \multirow{2}{*}{ Значение $C_{\text {trough }}$, мкГ/Мл } & \multicolumn{3}{|c|}{ ПФК ${ }_{24}$, мКг.ч/мЛ } & \multicolumn{3}{|c|}{$\Pi \Phi \mathrm{K}_{24} / \mathrm{M} \Pi \mathrm{K}>400$} \\
\hline & M & $\min$ & $\max$ & МПК 1 мкг/мл (\%) & МПК 1,5 мкг/мл (\%) & МПК 2 мкг/мл (\%) \\
\hline$<10$ & 395,1776 & 361,2053 & 421,9468 & 16 & 0 & 0 \\
\hline 10-15 & 517,7069 & 502,5894 & 564,0411 & 100 & 0 & 0 \\
\hline $15-20$ & 650,2483 & 578,911 & 721,5856 & 100 & 50 & 0 \\
\hline$>20$ & 783,8409 & 667,7073 & 910,8016 & 100 & 100 & 38 \\
\hline
\end{tabular}

от начала терапии достоверно отличались от значений по данным МM $(11,32(8,1-16,4)$ и 16,59 $(14,03-24,8)$ мкг/мл соответственно; $p=0,004)$.

Наши данные согласуются с результатами других исследований, в которых была отмечена высокая вариабельность параметров ФК, а также отношения ПФК ${ }_{24} / \mathrm{MПK}>400$ у пациентов в отделениях интенсивной терапии, которым проводили антибактериальную терапию ванкомицином в стандартных дозах $[15,16]$.

Различия в результатах ФКИ и ММ могут быть связаны с рядом недостатков большинства математических моделей. В однокомпартментных ММ используют в основном для расчетов фиксированные значения $V_{d}-$ среднюю величину 0,7 л/кг. По данным ФКИ вариабельность этого параметра составляет от 0,2 до 1,25 л/кг и зависит от объема циркулирующей крови, уровня альбумина и т. д. Для расчета $\mathrm{K}_{\text {еl }}$ используют значение KK по Кокрофту-Голту. В настоящее время не существует идеальной формулы для оценки скорости элиминации лекарственного препарата по уровню эндогенного креатинина $[17,18]$.

Ряд авторов отмечают в своих исследованиях, что метод применения стандартных номограмм или ММ для предсказания параметров ФК имеет ряд ограничений. Вопервых, большинство этих методов были валидированы на ограниченной популяции здоровых добровольцев или пациентов в стабильном состоянии. Во-вторых, целевые значения равновесных $C_{\text {trough }}$ рассматривались в пределах 5-10 мкг/мл. В настоящее время диапазон целевых концентраций вырос до 15-20 мкг/мл с учетом результатов микробиологических исследований $[19,20]$.

Другая актуальная тема для дискуссии - наличие необходимости достигать столь высоких значений $C_{\text {trouph }}$ и отношения ПФК $\mathrm{K}_{24} / \mathrm{MПK}$ на уровне 400 или выше. По данным локального микробиологического мониторинга при значениях МПК, равных 1 или < 1 мкг/Мл, достижение диапазона $C_{\text {trough }}$ 15-20 мкг/мл не обязательно [21].

В ходе ретроспективного исследования было показано, что более $30 \%$ пациентов достигали целевого отношения ПФК ${ }_{24} / \mathrm{M} \Pi$ > 400 даже при $C_{\text {trough }}$ ниже 15 мкг/Мл. По данным регрессивного анализа значение $C_{\text {trough }}$ на уровне 10,8 мкг/мл являлось предиктором достижения целевого отношения ПФК ${ }_{24} / \mathrm{M} К>400$ [22].
По данным нашего исследования у больных на фоне стандартных режимов дозирования ванкомицина была отмечена положительная клинико-лабораторная динамика, хотя значения $C_{\text {trough }}$ находились в пределах 10-15 мкг/мл (табл. 2). Достижение целевого отношения $П Ф \mathrm{~K}_{24} / \mathrm{MПК} \mathrm{>} 400$ можно объяснить результатами микробиологического мониторинга в нашем ЛПУ (S. aureus, МПК ванкомицина < 1 мкг/мл в 60-70\% случаев).

В ходе прогнозирования вероятности достижения целевого отношения ФК/ФД при повышении уровня МПК до 1,5 и 2 мкг/мл риск эффективности АБТ ванкомицином снижается в 30 и в 70\% случаев.

Получены данные, что индивидуальное изменение режимов дозирования с помощью ММ на основе результатов расширенного ФКИ $\left(C_{\text {trough, }}, C_{\text {peak }}, П Ф \mathrm{~K}_{24}\right)$ имело преимущество по сравнению с режимом дозирования по данным мониторирования $C_{\text {trough }}$ [23].

Результаты ФКИ для особых групп пациентов необходимы для разработки ММ параметров ФК и определения оптимальных режимов дозирования ванкомицина. Данные этих исследований служат основой для создания популяционных математических моделей, что требует увеличения количества ФКИ у пациентов в различных когортах [24, 25].

\section{ВЫВОДЫ}

Результаты нашего исследования свидетельствуют о высокой вариабельности параметров ФК ванкомицина, полученных различными методами. Различия параметров ФК по данным ФКИ и ММ подтверждают необходимость ТЛМ у пациентов с нарушениями функции почек в послеоперационном периоде. Антибактериальная терапия ванкомицином с учетом $C_{\text {trough }}$ по данным ТЛМ позволяет повысить безопасность терапии у больных с острыми нарушениями функции почек. Эффективность антибактериальной терапии ванкомицином ограничена чувствительностью возбудителя (МПК) и требует расчета отношения ПФК ${ }_{24} / \mathrm{M} К$. Необходимо продолжить ФКИ ванкомицина у больных с острыми нарушениями функции почек в послеоперационном периоде для усовершенствования методики ММ в представленной когорте. 


\section{Литература}

1. Rybak M, Lomaestro B, Rotschafer JC, Moellering R, Craig W, Billeter $\mathrm{M}$, et al. Therapeutic monitoring of vancomycin in adult patients: a consensus review of the American Society of HealthSystem Pharmacists, the Infectious Diseases Society of America, and the Society of Infectious Diseases. Am J Health Syst Pharm. 2009 Jan 1; 66 (1): 82-98.

2. Ye ZK, et al. Therapeutic drug monitoring of vancomycin: a guideline of the Division of Therapeutic Drug Monitoring. J Antimicrob Chemother. 2016 Nov 11; 71 (11): 3020-25.

3. Бондарева И. Б. Программное обеспечение для анализа данных ФК/ФД исследований. Клиническая фармакокинетика. 2005; 2 (3): 9-13.

4. Brendel K, Dartois C, Comets E, et al. Are population pharmacokinetic and/or pharmacodynamic models adequately evaluated? A survey of the literature from 2002 to 2004. Clin Pharmacokinet. 2007; 46 (3): 221-34.

5. Clinical Calculators [интернет]. Available from: http://clincalc. com/Nancomycin

6. Lake KD, Peterson CD. A simplified dosing method for initiating vancomycin therapy. Pharmacotherapy. 1985 Nov-Dec; 5 (6): 340-44.

7. Al-Kofide H, Zaghloul I, Al-Naim L. Pharmacokinetics of vancomycin in adult cancer patients. J Oncol Pharm Pract. 2010 Dec 16; 16(4): 245-250.

8. Burton ME, Gentle DL, Vasko MR. Evaluation of a Bayesian method for predicting vancomycin dosing. DICP. 1989; 23 (4): 294-300.

9. Khwaja A. KDIGO clinical practice guidelines for acute kidney injury. Nephron Clin Pract. 2012; 120 (4): 179-84.

10. Инструкция по медицинскому применению [интернет]. Доступно по ссылке: http://grls.rosminzdrav.ru/Grls_View_v2.aspx?routingGuid= 80390ffo-c656-4e1b-a33e-a30232cccf1d\&t=

11. Hammett-Stabler CA, Johns T. Laboratory guidelines for monitoring of antimicrobial drugs. Clin Chem. 1998 May; 44 (5): 1129-40

12. Core Team. R: A language and environment for statistical computing. R Foundation for Statistical Computing, Vienna, Austria. 2017 Available from: https://www.R-project.org

13. Bauer LA. Applied clinical pharmacokinetics. New York: McGrawHill, 2001. p. 26-49.

14. Matzke GR, McGory RW, Halstenson CE, Keane WF. Pharmacokinetics of vancomycin in patients with various degrees of renal function. Antimicrob Agents Chemother. 1984 Apr; 25 (4): 433-7.

\section{References}

1. Rybak M, Lomaestro B, Rotschafer JC, Moellering R, Craig W, Billeter $\mathrm{M}$, et al. Therapeutic monitoring of vancomycin in adult patients: a consensus review of the American Society of HealthSystem Pharmacists, the Infectious Diseases Society of America, and the Society of Infectious Diseases. Am J Health Syst Pharm. 2009 Jan 1; 66 (1): 82-98.

2. Ye ZK, et al. Therapeutic drug monitoring of vancomycin: a guideline of the Division of Therapeutic Drug Monitoring. J Antimicrob Chemother. 2016 Nov 11; 71 (11): 3020-25.

3. Bondareva I. B. Programmnoe obespechenie dlya analiza dannyh FK/FD issledovanij. Klinicheskaya farmakokinetika. 2005; 2 (3): 9-13.

4. Brendel K, Dartois C, Comets E, et al. Are population pharmacokinetic and/or pharmacodynamic models adequately evaluated? A survey of the literature from 2002 to 2004. Clin Pharmacokinet. 2007; 46 (3): 221-34.

5. Clinical Calculators [internet]. Available from: http://clincalc.com/ Vancomycin.

6. Lake KD, Peterson CD. A simplified dosing method for initiating vancomycin therapy. Pharmacotherapy. 1985 Nov-Dec; 5 (6): 340-44.
15. Patel N, Pai MP, Rodvold KA, Lomaestro B, Drusano GL, Lodise TP. Vancomycin: we can't get there from here. Clin Infect Dis. 2011 Apr 15; 52 (8): 969-74.

16. Bel KA, Bourguignon L, Marcos M, Ducher M, Goutelle S. Is trough concentration of vancomycin predictive of the area under the curve? A clinical study in elderly patients. Ther Drug Monit. 2017 Feb; 39 (1): 83-7.

17. Moise-Broder PA, Forrest A, Birmingham $\mathrm{MC}$, et al Pharmacodynamics of vancomycin and other antimicrobials in patients with Staphylococcus aureus lower respiratory tract infections. Clin Pharmacokinet. 2004; 43 (13): 925-42.

18. Paglialunga S, Offman E, Ichhpurani N, Marbury TC, Morimoto BH. Update and trends on pharmacokinetic studies in patients with impaired renal function: practical insight into application of the FDA and EMA guidelines. Expert review of clinical pharmacology. 2017; 10 (3): 273-83.

19. Prybylski JP. Vancomycin Trough Concentration as a Predictor of Clinical Outcomes in Patients with Staphylococcus aureus Bacteremia: A Meta-analysis of Observational Studies. Pharmacotherapy. 2015 Oct; 35 (10): 889-98.

20. Murphy JE, Gillespie DE, Bateman CV. Predictability of vancomycin trough concentrations using seven approaches for estimating pharmacokinetic parameters. Am J Health Syst Pharm. 2006 Dec 1; 63 (23): 2365-70.

21. del Mar Fernández de Gatta Garcia M, Revilla N, Calvo MV Domínguez-Gil A, Sánchez Navarro A. Pharmacokinetic/ pharmacodynamic analysis of vancomycin in ICU patients. Intensive Care Med. 2007 Feb; 33 (2): 279-85.

22. Neely MN, Youn G, Jones B, Jelliffe RW, Drusano GL, Rodvold $\mathrm{KA}$, et al. Are vancomycin trough concentrations adequate for optimal dosing? Antimicrob Agents Chemother. 2014; 58 (1): $309-16$.

23. Pai MP, Neely M, Rodvold KA, Lodise TP. Innovative approaches to optimizing the delivery of vancomycin in individual patients. Adv Drug Deliv Rev. 2014 Nov 20; 77: 50-7.

24. Purwonugroho TA, Chulavatnatol $S$, Preechagoon $Y$, Chindavijak B, Malathum K, Bunuparadah P. Population pharmacokinetics of vancomycin in Thai patients. The Scientific World Journal. 2012;2012:762649. DOI: 10.1100/2012/762649.

25. Zalloum N, Saleh Ml, Al Haj M, Balbisi M, Al-Ghazawi M. Population pharmacokinetics of vancomycin in Jordanian patients Tropical Journal of Pharmaceutical Research. 2018; 17 (2): 351-58.

7. Al-Kofide $\mathrm{H}$, Zaghloul I, Al-Naim L. Pharmacokinetics of vancomycin in adult cancer patients. J Oncol Pharm Pract. 2010 Dec 16; 16 (4): 245-250.

8. Burton ME, Gentle DL, Vasko MR. Evaluation of a Bayesian method for predicting vancomycin dosing. DICP. 1989; 23 (4): 294-300.

9. Khwaja A. KDIGO clinical practice guidelines for acute kidney injury. Nephron Clin Pract. 2012; 120 (4): 179-84.

10. Instrukciya po medicinskomu primeneniyu [internet]. Available from: http://grls.rosminzdrav.ru/Grls_View_v2.aspx?routingGuid= 80390ffo-c656-4e1b-a33e-a30232cccf1d\&t=.

11. Hammett-Stabler CA, Johns T. Laboratory guidelines for monitoring of antimicrobial drugs. Clin Chem. 1998 May; 44 (5): $1129-40$

12. Core Team. R: A language and environment for statistical computing. R Foundation for Statistical Computing, Vienna, Austria. 2017 Available from: https://www.R-project.org.

13. Bauer LA. Applied clinical pharmacokinetics. New York: McGrawHill, 2001.p. 26-49.

14. Matzke GR, McGory RW, Halstenson CE, Keane WF. Pharmacokinetics of vancomycin in patients with various 
degrees of renal function. Antimicrob Agents Chemother. 1984 Apr;25(4):433-7.

15. Patel N, Pai MP, Rodvold KA, Lomaestro B, Drusano GL, Lodise TP. Vancomycin: we can't get there from here. Clin Infect Dis. 2011 Apr 15; 52 (8): 969-74.

16. Bel KA, Bourguignon L, Marcos M, Ducher M, Goutelle $\mathrm{S}$. Is trough concentration of vancomycin predictive of the area under the curve? A clinical study in elderly patients. Ther Drug Monit. 2017 Feb; 39 (1): 83-7.

17. Moise-Broder PA, Forrest $\mathrm{A}$, Birmingham $\mathrm{MC}$, et al. Pharmacodynamics of vancomycin and other antimicrobials in patients with Staphylococcus aureus lower respiratory tract infections. Clin Pharmacokinet. 2004; 43 (13): 925-42.

18. Paglialunga S, Offman E, Ichhpurani N, Marbury TC, Morimoto BH. Update and trends on pharmacokinetic studies in patients with impaired renal function: practical insight into application of the FDA and EMA guidelines. Expert review of clinical pharmacology. 2017; 10 (3): 273-83.

19. Prybylski JP. Vancomycin Trough Concentration as a Predictor of Clinical Outcomes in Patients with Staphylococcus aureus Bacteremia: A Meta-analysis of Observational Studies. Pharmacotherapy. 2015 Oct; 35 (10): 889-98.
20. Murphy JE, Gillespie DE, Bateman CV. Predictability of vancomycin trough concentrations using seven approaches for estimating pharmacokinetic parameters. Am J Health Syst Pharm. 2006 Dec 1; 63 (23): 2365-70.

21. del Mar Fernández de Gatta Garcia M, Revilla N, Calvo MV, Domínguez-Gil A, Sánchez Navarro A. Pharmacokinetic/ pharmacodynamic analysis of vancomycin in ICU patients. Intensive Care Med. 2007 Feb; 33 (2): 279-85.

22. Neely MN, Youn G, Jones B, Jelliffe RW, Drusano GL, Rodvold KA, et al. Are vancomycin trough concentrations adequate for optimal dosing? Antimicrob Agents Chemother. 2014; 58 (1): 309-16.

23. Pai MP, Neely M, Rodvold KA, Lodise TP. Innovative approaches to optimizing the delivery of vancomycin in individual patients. Adv Drug Deliv Rev. 2014 Nov 20;77:50-7.

24. Purwonugroho TA, Chulavatnatol S, Preechagoon Y, Chindavijak B, Malathum K, Bunuparadah P. Population pharmacokinetics of vancomycin in Thai patients. The Scientific World Journal. 2012;2012:762649. DOI: 10.1100/2012/762649.

25. Zalloum N, Saleh Ml, Al Haj M, Balbisi M, Al-Ghazawi M. Population pharmacokinetics of vancomycin in Jordanian patients Tropical Journal of Pharmaceutical Research. 2018; 17 (2): 351-58. 